\title{
La biblioteca universitaria ante el nuevo modelo social y educativo
}

\author{
Por Luisa Orera-Orera
}

Resumen: El concepto de biblioteca debe ser revisado periódicamente, cuando en su proceso evolutivo se producen cambios transcendentales. Ahora nos encontramos en una de esas fases. Por ello, en este artículo se define el nuevo modelo de biblioteca universitaria que ha surgido para responder a dos tipos de cambios: los que la sociedad de la información requiere y los que son necesarios para desarrollar el Espacio Europeo de Educación Superior (EEES). De acuerdo con estas exigencias, se va perfilando un nuevo modelo de biblioteca universitaria híbrida que basa su gestión en la filosofía de la globalización, el uso de las nuevas tecnologías de la información y comunicación

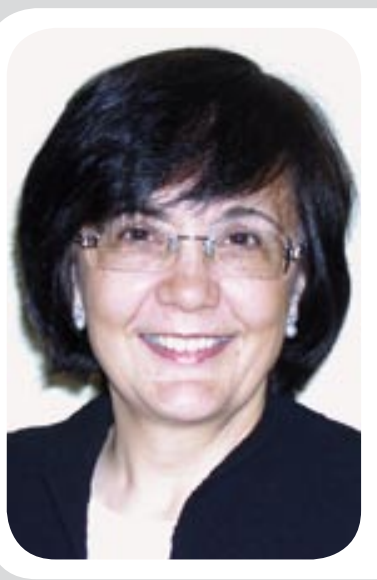

Luisa Orera-Orera, profesora titular de biblioteconomía de la Universidad de Zaragoza, es miembro del Departamento de Ciencias de la Documentación e Historia de la Ciencia. Imparte docencia en la Facultad de Filosofía de la Universidad de Zaragoza. Ha desarrollado una amplia actividad profesional a través de su labor docente en la Universidad de Zaragoza y otras universidades a las que ha sido invitada, así como a través de su labor investigadora. Actualmente es Miembro del Comité científico de la Revista Interamericana de Bibliotecología y Presidenta del Comité Nacional de Evaluación para las bibliotecas universitarias españolas, de la Agencia Nacional de Calidad y Acreditación (Aneca).

(TICs), en la cooperación y en la calidad de sus servicios.

Palabras clave: Concepto de biblioteca, Definición de biblioteca, Biblioteca universitaria, Biblioteca híbrida, Espacio Europeo de Educación Superior (EEES), Colecciones bibliotecarias.

\section{Title: The university library in the context of the new social and educational model}

Abstract: The concept of a library must be revised periodically, whenever the evolutionary process produces a truly transcendental change. The present is one such point in time. A new model for a university library is described, one that has emerged in response to two types of changes: those required by the information society and those needed for the development of a European Space for Higher Education (ESHE). In keeping with these requirements, a hybrid university library is now developing, whose management is based on the philosophy of globalization, the use of new information and communication technologies (ICTs), cooperation, and the quality of services provided.

Keywords: Library concept, Library definition, Academic library, Hybrid library, European Higher Education Area (EHEA), Library resources.

Orera-Orera, Luisa. "La biblioteca universitaria ante el nuevo modelo social y educativo". En: El profesional de la información, 2007, julio-agosto, v. 16, n. 4, pp. 329-337.

DOI: 10.3145/epi.2007.jul.07

\section{Introducción}

EL CONCEPTO DE BIBLIOTECA DEBE SER REVISADO periódicamente cuando en su proceso evolutivo se producen cambios transcendentales. Ahora nos encontramos en uno de esos momentos, por lo que trataremos de exponer la idea de biblioteca en el nuevo contexto.

Para ello hemos elegido la biblioteca universitaria, por conside- rar que es en este tipo de centro en el que se proyectan con gran fuerza dichos cambios. En su caso, éstos han de situarse en dos escenarios relacionados entre sí: el social y el universitario.

En cuanto al primero, es preciso señalar que nos encontramos ante una revolución comparable en magnitud a otras anteriores como la revolución agrícola o la industrial y cuyas consecuencias todavía no alcanzamos a valorar en su justa medida. A partir de ella, ha surgido un nuevo modelo social, la denominada sociedad de la información y del conocimiento, que se identifica por una serie de características tales como: la importancia y el valor económico de la información; el peso de las tecnologías de la información y de la comunicación (cuyo exponente máximo es sin duda internet), y el fenómeno de la globalización.

En este contexto, la biblioteca se enfrenta a nuevos retos tales como 
los relacionados con la gestión de la información digital, la adaptación de los servicios tradicionales a las nuevas necesidades, o los problemas que surgen entre el derecho de autor, defendido sobre todo por los editores, y el de los ciudadanos a acceder a la información. Este escenario ha traído un nuevo modelo que de forma general se conoce como biblioteca híbrida (Orera, 2005), en el que se conjugan elementos nuevos y otros tradicionales que permiten seguir hablando de la continuidad de la biblioteca, a pesar de los cambios.

Por otra parte, no hay que olvidar que la biblioteca universitaria es una pieza clave de la universidad, el segundo escenario al que nos referíamos. En Europa, la universidad se halla inmersa en un cambio profundo que deberá desembocar en la creación del denominado Espacio Europeo de Educación Superior (EEES), no más tarde de 2010, tal y como establece la Declaración de Bolonia. En cuanto a la biblioteca universitaria se refiere, este cambio afectará a las funciones que desempeña dentro de la universidad, donde habrá de ejercer un papel más activo y lograr una mayor integración.

En los apartados siguientes de este artículo, trataremos de exponer las principales transformaciones a las que se enfrentan las bibliotecas universitarias en ambos contextos: el social y el universitario, para así definir el nuevo modelo y sus características.

La síntesis que aquí presentamos es fruto de una reflexión basada en el análisis de diversas publicaciones científicas así como el de algunas bibliotecas universitarias a través de sus sitios web o de la observación directa del funcionamiento de algunas de ellas.

\section{Un nuevo modelo de biblioteca para un nuevo modelo de sociedad: la biblioteca híbrida}

Como ya hemos señalado, el estudio de la biblioteca universitaria requiere hacer referencia al contexto bibliotecario general y dentro de él, al margen de la tipología bibliotecaria, es preciso reiterar que estamos asistiendo al nacimiento de un nuevo modelo: la biblioteca denominada híbrida, al que se ha llegado a través de un largo proceso evolutivo.

Al encontrarnos inmersos en su proceso de desarrollo, carecemos quizá de perspectiva suficiente para juzgar su trascendencia. Tal vez, como han dicho algunos, sólo se trate de una etapa de transición hacia una biblioteca totalmente digital. En todo caso, independientemente del futuro que le espere, la biblioteca híbrida es la realidad que viven ahora mismo los centros bibliotecarios. Por otra parte, lo que sí puede afirmarse es que los hechos han desmentido por el momento a aquellos que veían en internet y en el desarrollo de la información digital el final de las bibliotecas (Herring, 2001).

La biblioteca híbrida es la suma de los elementos tradicionales de la biblioteca con otros nuevos que derivan de la importancia de la información digital, de las nuevas tecnologías y de la telemática. Es una entidad mixta donde conviven documentos tradicionales con in-

\footnotetext{
"La biblioteca híbrida es la suma de los elementos tradicionales de la biblioteca con otros nuevos que derivan de la importancia de la información digital, de las nuevas tecnologías y de la telemática"
}

formación digital y servicios que se dan en sus dependencias físicas con otros que se ofrecen a través de internet. En ella se atiende a usuarios presenciales y remotos. Es la suma de lo real y lo virtual. A la hora de fijar este concepto de centro híbrido, son muchos los aspectos que resultan relevantes:

- El nuevo papel del bibliotecario, cuya importancia es cada vez mayor debido a la desmesurada cantidad de información existente y la necesidad de seleccionarla con criterios de calidad. La gestión de la información requiere plantillas más abultadas con formación diversificada, en las que el personal con conocimientos informáticos es cada día más imprescindible.

Además, la gestión de la biblioteca híbrida exige una formación cada vez más cualificada y continua para hacer frente a los cambios que se producen en la sociedad de la información.

En España, es la universidad la que se encarga de formar al personal bibliotecario, impartiéndose los tres ciclos que actualmente integran los estudios universitarios: diplomatura de biblioteconomía y documentación; licenciatura en documentación y doctorado. Al igual que el resto de las carreras universitarias, estos estudios se encuentran inmersos en un proceso de cambio para adaptarse a las exigencias del EEES (Orera, 2002). En cuanto a la formación continua, también desempeñan un importante papel las asociaciones profesionales del sector.

- El nuevo modelo de gestión sustentado en la informática y las telecomunicaciones. Internet y el uso de tecnología web se hacen imprescindibles. El uso del correo electrónico es básico en la prestación de los servicios bibliotecarios, haciéndolos más inmediatos y flexibles.

Junto a las ventajas, el uso de las nuevas tecnologías también 
conlleva inconvenientes. Por ejemplo, la importante base tecnológica sobre la que se asienta la nueva biblioteca hace que su mantenimiento sea caro, con lo se corre el peligro de que la "brecha digital" aumente, ante la imposibilidad de renovación de equipos informáticos por parte, sobre todo, de los países más pobres. El uso de las nuevas tecnologías presenta también otros problemas todavía no resueltos como: las deficiencias en los soportes físicos y en las telecomunicaciones; la definición de normas informáticas; los relacionados con el diseño de interfaces, etc. (García Camarero, 2001, p. 17). En este área se abre un gran campo de trabajo para buscar soluciones a la pervivencia de la biblioteca como un servicio para todos.

- La ya aludida transformación que están experimentando los servicios de la biblioteca, tanto a los ofrecidos en sus locales, como a distancia, a través de visitas virtuales, convirtiéndose estos últimos en atemporales. Al ser accesibles desde cualquier punto donde se disponga de un ordenador conectado a internet, los usuarios se multiplican $\mathrm{y}$ diversifican. Al respecto, cada vez son más los que piensan que, andando el tiempo, los más numerosos serán precisamente los que se conectan a través de la Red (Pérez, 1998, p. 181).

En el nuevo contexto, la formación de usuarios se torna decisiva y amplía su campo de acción. añadiendo a su objetivo tradicional, que se centraba en formar al usuario en el uso de la biblioteca, otros como la formación en el uso de las nuevas tecnologías, de los programas informáticos, y en definitiva, de la información en general.

- También el proceso técnico al que se someten los documentos en la biblioteca con el fin de lograr recuperarlos posteriormente, está experimentando cambios importantes. Y así, junto a técnicas de tratamiento para documentos tradicionales como la catalogación, la presencia cada vez más importante de información digital en las redes, hace necesarias otras nuevas como los metadatos.

- La cooperación interbibliotecaria no ha nacido con la biblioteca híbrida, pero en el futuro está llamada a incrementarse. La complejidad del mundo de la información, las cada vez más variadas necesidades de información de los usuarios, los reducidos presupuestos de que a veces disponen las bibliotecas, unido a las grandes posibilidades que ofrecen las nuevas tecnologías, están dando lugar a que cada vez más, las bibliotecas en general y las universitarias en particular, orienten sus políticas hacia la cooperación. En realidad, la gestión de una biblioteca universitaria es hoy imposible si no se basa en la cooperación. Por otra parte, el nuevo escenario de la biblioteca híbrida, con un peso cada vez mayor de los recursos digitales en sus colecciones, ha traído nuevas formas de cooperación como los consorcios (Giordano, 2000).

Ante la imposibilidad de recoger aquí todos los elementos propios de la biblioteca híbrida, hemos seleccionado para referirnos de forma más pormenorizada a uno que consideramos clave para identificarla, como es el concepto de colección.

1. Un nuevo concepto de colección.

La colección constituye el elemento fundamental de cualquier tipo de biblioteca. Analizarla supone en realidad estudiar la biblioteca, ya que es el elemento en el que se ba- san los principales servicios dados a los usuarios. Antes del proceso de cambio que afecta a la biblioteca, se definía la colección bibliotecaria como un conjunto de documentos previamente seleccionados, que se adquiría y trataba para ponerlo a disposición de sus usuarios.

Con la avalancha de información digital fácilmente accesible a través de un terminal de ordenador conectado a la Red, se empezó a cuestionar el futuro de las bibliotecas. ¿Cuál sería su papel si existía ya gran cantidad de información de tan fácil acceso para todo el mundo? Las visiones más apocalípticas vieron el final de los centros bibliotecarios.

En este nuevo escenario, se concibieron como excluyentes las colecciones tradicionales de las bibliotecas y la información digital disponible en línea, formulándose la contraposición de ambas realidades como un debate entre: propiedad o acceso. La función de las bibliotecas ya no estaba en transferir información a través de la creación de colecciones propias, sino en facilitar el acceso a la misma. Por otra parte y en contra del desarrollo de colecciones tradicionales, se señalaron una serie de problemas como: los escasos presupuestos de las bibliotecas frente a los altos costes de las publicaciones; los relacionados con su almacenamiento, conservación, descarte, evaluación de colecciones, adquisición de información irrelevante, duplicada, etc. Todo ello llevó a numerosos autores a augurar el fin de dichas colecciones (Pérez, 1998, pp. 178-179).

Sin embargo, el paso del tiempo ha demostrado que por el momento,

\section{"La importante base tecnológica sobre la que se asienta la nueva biblioteca hace que su mantenimiento sea caro, con lo se corre el peligro de que la 'brecha digital' aumente"}


los negros augurios no se han cumplido porque la información digital plantea todavía algunas incógnitas. Son ya clásicas las 10 razones por las que internet no puede sustituir a las bibliotecas (Herring, 2001). De ellas destacamos algunas: no todo se encuentra en la Red; no todo lo que contiene puede encontrarse; no existe un control de calidad, etc. Otra incertidumbre la constituye el problema de su permanencia. Y esto por causas tales como el desconocimiento que existe respecto a su estabilidad y duración, la falta de seguridad de que los editores de productos digitales los conserven, la necesidad de convertir formatos cada vez que se vuelvan obsoletos, etc. (Pérez, 1998, p. 180).

Por otra parte, los usuarios de colecciones de las bibliotecas universitarias tienen necesidades y posibilidades diferentes. Hay colecciones básicas, sobre todo de apoyo a la docencia, a las cuales se debe garantizar su permanencia y accesibilidad para todos y de forma permanente.

La progresiva consolidación de colecciones mixtas ha dado lugar a una postura más realista: ambas tipologías son complementarias y necesarias en las bibliotecas. Ya no hablamos sólo de un conjunto selectivo de documentos que se poseen y se hacen accesibles a los usuarios, sino que por colección también debe entenderse aquellos recursos informativos que la biblioteca, una vez seleccionados, pone a disposición de los usuarios, sin necesidad de que sean de su propiedad. Así, el formato no es lo importante, sino la facilidad o dificultad con la que se accede a la información (Pérez, 1998, p. 187). Esta postura conciliadora resulta lógica si tenemos en cuenta además, que la permeabilidad entre documentos impresos y digitales es total: un documento en papel puede convertirse en digital a través de un escáner y uno digital puede pasar a papel a través de la impresora.
De acuerdo con lo anterior, podemos afirmar que el principal cambio que han experimentado las colecciones bibliotecarias ha consistido en su evolución hacia colecciones híbridas, constituidas por documentos de los que la biblioteca dispone físicamente, y por información digital, residente o no en servidores propios y que ha seleccionado y ofrece a los usuarios. Este carácter dual de la colección es precisamente lo que fundamenta el cambio de modelo de biblioteca al que nos estamos refiriendo.

Por otra parte, para acabar de definir el concepto de colección bibliotecaria, es preciso aludir también a los métodos de incorporación de documentos a la misma. Antes de la aparición de la documentación digital, los procedimientos clásicos de adquisición en las bibliotecas universitarias eran: la compra, el canje y las donaciones. La aparición de documentos digitales en soportes como el cd-rom no alteraron substancialmente la situación. En ambos casos se trataba de la adquisición de objetos físicos propiedad de la biblioteca y que los usuarios podían usar tantas veces como fuera necesario, siempre que se observaran las condiciones de respeto a la propiedad intelectual, haciéndose un uso correcto de la información. Estos procedimientos y el régimen de explotación de documentos se mantienen actualmente para la parte de la colección que podemos denominar tradicional.

El cambio se ha producido con la llegada de la información digital accesible a través de internet y muy especialmente con el desarrollo de la tecnología web. Los mecanismos de adquisición tradicionales no son aplicables porque las circunstancias han cambiado. La biblioteca, al adquirir este tipo de recursos, no dispone de documentos físicos que guarda en sus dependencias, sino que muchas veces lo que compra es el derecho a acceder a materiales que residen incluso en servidores ajenos, a través de suscripciones o licencias en las que se fijan las condiciones de acceso. La biblioteca ha dejado de ser propietaria de una parte de sus colecciones.

Hay que decir además que, propiciadas por las posibilidades que ofrecen las nuevas tecnologías, las condiciones de acceso a los documentos no son homogéneas y que el futuro no está todavía definido. Día a día aparecen nuevas soluciones que tratan de satisfacer a la carta nuevas necesidades. Ahora ya es posible incluso comprar solamente aquella parte del documento que interesa, sin necesidad de adquirirlo completo. Un ejemplo de esta modalidad podemos encontrarlo en la plataforma e-Libro.

Tampoco los problemas relacionados con el acceso son comunes a todos los documentos. En el caso de las bibliotecas universitarias es el acceso a las revistas comerciales el que representa mayores dificultades económicas a causa de su enorme crecimiento y de los precios cada vez más altos impuestos sobre todo por las grandes editoriales que monopolizan el sector: Reed Elsevier; Springer; Wolters Kluwer, etc.

\section{"La edición digital supone la reducción de costes tanto en la producción como en la distribución de documentos, por lo que se pensaba que iba a favorecer a las bibliotecas"}

La edición digital supone la reducción de costes tanto en la producción como en la distribución de documentos, por lo que se pensaba que iba a favorecer a las bibliotecas. Sin embargo, en el nuevo escenario han surgido otros factores que no 
sólo han neutralizado las ventajas anteriores, sino que en ocasiones han agravado los costes de las adquisiciones. Nos referimos, por una parte, a la extensión de la piratería y por otra, a los abusos cometidos por algunos distribuidores a la hora de fijar precios. Además, entre los editores y distribuidores comerciales de información digital se ha ido abriendo paso la idea, inspirada en otros medios como la televisión, de cobrar de acuerdo con su uso.

Los altos costes que para las bibliotecas universitarias supone el acceso a las revistas, les han llevado a tratar de conseguir condiciones ventajosas a través de la creación de nuevas formas de cooperación como los consorcios.

Pero además de los consorcios han aparecido otras posibilidades. Junto a los altos costes, la facilidad del proceso de la edición digital, la paradoja que supone que las universidades tengan que dedicar importantes presupuestos para acceder a las revistas cuando muchas veces son sus investigadores los autores de esos contenidos y por último, los cambios que están teniendo lugar en el proceso mediante el que se genera el conocimiento científico así como en el proceso de su comunicación, que requiere una mayor celeridad para lograr mayor eficacia, han propiciado el nacimiento de un movimiento internacional, cada vez más fuerte, en favor de fomentar la publicación de los trabajos científicos por los propios investigadores, llegar a acuerdos razonables con los editores y en definitiva, crear unas condiciones en las que cada vez resulte más fácil el acceso libre a la información científica. Sin duda alguna, en el futuro este movimiento va a tener consecuencias importantes en el modelo de comunicación y acceso a la información científica. En este contexto están apareciendo iniciativas con origen y características diversas como, por ejemplo: Sparc (Scholary Publishing and Academic Resource Coalition); PubMed Central; el proyecto $E$ Biosci; E-LIS (E-prints in Library and Information Science), etc. En este marco, la biblioteca universitaria está llamada a desempeñar una nueva función emergente en el proceso de edición de documentos y la creación de bibliotecas digitales que contribuyan a enriquecer las colecciones tradicionales.

Ejemplo de lo que decimos son los proyectos de digitalización de fondos antiguos, propiciados por una serie de causas como la voluntad de difundir colecciones valiosas evitando a la vez su deterioro, su carácter atractivo, que suelen atraer con facilidad la colaboración financiera de empresas, etc.

Junto a las bibliotecas digitales de fondo antiguo, cada día son más frecuentes las creadas con el fin de apoyar el proceso de aprendizaje y autoformación de los estudiantes, lo que constituye la base del modelo de enseñanza hacia el que caminan las universidades europeas, sin olvidar la importancia que en este marco va a tener la educación a distancia. Las bibliotecas, junto con los profesores, van a jugar un papel decisivo a la hora de hacer accesibles estos recursos a los estudiantes; proyectos como Cerise o Jurisguide, así lo prueban (Neouze, 2002, p. 351).

Lo mismo sucede con las colecciones digitales relacionadas con la investigación, en las que las bibliotecas digitales de tesis ocupan un destacado lugar. A través de ellas puede hacerse visible una parte importante de la investigación que se realiza en las universidades. Las ventajas que ofrecen este tipo de recursos están logrando que hoy día, en todo el mundo, sean muy numerosas las universidades embarcadas en estos proyectos (Orera, 2003).

\section{Cambios en el contexto universitario: el Espacio Europeo de Educación Superior (EEES)}

El desarrollo armónico de los distintos sistemas nacionales europeos de educación superior que requiere la implantación del EEES pretende lograrse antes de 2010

\title{
Suscripciones
}

\author{
Renovar (o comenzar) la suscripción a "El profesional de la información" \\ es ágil y sencillo.
}

Usted puede gestionar online su suscripción conectándose a esta web: http://www.elprofesionaldelainformacion.com/suscripciones.html

Si lo desea puede comunicar con nosotros dirigiéndose a: suscripciones@elprofesionaldelainformacion.com 
a través de la adopción de una serie de elementos comunes como la existencia de dos niveles en las titulaciones y la implantación de un sistema de semestres y de créditos ECTS (European Credits Transfer System), entre otros.

De todos los elementos comunes en los que se sustenta, es preciso referirse de forma más pormenorizada al principio de calidad, ya que éste lo impregna todo. La construcción del EEES basa su razón de ser en la búsqueda de la misma, al querer constituirse en un referente mundial en lo que a educación superior se refiere. Además, son necesarios unos mínimos de calidad que hagan viable el intercambio entre los componentes del sistema.

La búsqueda de calidad así como la preocupación por hacerla patente con el fin de dar a conocer el prestigio de las universidades hace necesaria su medición; es decir, lleva implícito un proceso de evaluación. El modelo que se ha ido gestando en Europa en estos últimos años es una evaluación institucional, que analiza cada uno de los elementos necesarios para que la institución logre sus fines, recursos y resultados, pero atendiendo siempre al funcionamiento del conjunto. Este modelo se lleva a cabo mediante tres elementos fundamentales: La autoevaluación, realizada por una comisión formada por personas pertenecientes a la institución y que representa los distintos estamentos universitarios; la externa, llevada a cabo por un comité de expertos, y el informe final.

La calidad y su evaluación supone tomar como punto de referencia un modelo de universidad, que puede definirse teniendo en cuenta sus objetivos (De Miguel, 2001, pp. 387-396): transmisión de la cultura, ejercicio de la docencia y de la investigación científica, socialización de sus estudiantes y compromiso social.

\section{El nuevo modelo de univer- sidad}

Definirlo requiere referirse al menos a dos elementos clave: la misión docente y la investigadora.

La misión docente de la universidad se halla actualmente en un proceso de adaptación a las nuevas exigencias que la sociedad del conocimiento requiere. Simplificando, estos nuevos requisitos podemos reducirlos a dos: un nuevo sistema de aprendizaje y la implantación generalizada de las tecnologías de la información y comunicación (TICs).

El nuevo sistema de aprendizaje se basa en la necesidad de que los ciudadanos dispongan de una formación crítica que les capacite para interpretar una información que cambia continuamente y para que sean capaces de generar conocimiento. Esto no es posible si no se da al alumno universitario una formación centrada en el propio aprendizaje, proceso en el cual el profesor debe desempeñar un importante papel como agente activo en la motivación del alumno, en la mejora de la calidad de la docencia y en la innovación.

El citado sistema de créditos ECTS que ha sido adoptado por los países de la Unión Europea para estructurar los currículos universitarios, es un claro exponente de este cambio de concepción que se está operando en la educación superior. A diferencia del actual crédito utilizado hasta ahora en España, definido en función de la carga docente teórica y práctica impartida por el profesor, el nuevo modelo tiene en cuenta además el esfuerzo realizado por el alumno para la preparación de la asignatura y la realización de exámenes. Supone un cambio radical en la concepción del sistema educativo, al tener en cuenta el aprendizaje del estudiante. Desde el punto de vista del profesor, el concepto de carga docente se sustituye por el de actividad académica, que engloba además de las horas lectivas, el tiempo dedicado a organizar y supervisar el trabajo de los alumnos, la preparación de los exámenes, así como la de materiales didácticos de apoyo al aprendizaje (Moscoso).

Por otra parte, el nuevo modelo de sociedad requiere una formación continua para poder afrontar los cambios vertiginosos que en ella se producen. En este contexto también la universidad está llamada a ejercer un destacado papel a través de programas específicos y de la preparación de formadores que ejerzan su labor en otros niveles docentes diferentes al universitario. Más específicamente, también corresponde a la universidad la formación en nuevas tecnologías, para lograr la capacitación en las mismas de toda la sociedad y evitar así uno de los nuevos problemas con los que ésta se enfrenta actualmente: la brecha digital, a consecuencia de la cual, amplias capas de la población pueden quedar excluidas del acceso a la cultura.

El segundo motor del cambio del sistema docente lo constituyen las TICs, que deben ser la base de una formación universitaria de calidad y cuyo manejo es necesario tanto por parte del alumno como del profesor. La introducción de las mismas va a producir una serie de cambios substanciales que pueden resumirse en la crisis del sistema docente tradicional que se concentraba en el aula, favoreciendo la globalización de la enseñanza universitaria, la apertura de la universidad a colectivos que hasta ahora no habían tenido acceso y la formación continua a lo largo de la vida. Todo lo cual está produciendo un importante cambio en la concepción de la formación académica.

Desde hace más de un siglo, la investigación ha sido, junto con la docencia, la misión más importante de la universidad. De hecho, en algunos países europeos la uni- 
versidad es la responsable del $80 \%$ de la investigación que se realiza y emplea a la mayoría de los investigadores, por lo que su papel en la construcción del Espacio Europeo de Investigación es fundamental (Moscoso).

Como la docencia, también el modelo investigador está cambiando, propiciando la aparición de la denominada universidad investigadora (De Miguel, 2001), que se basa en la unión de la docencia y de la investigación y donde los profesores realizan a la vez ambas tareas. Así, no sólo se avanza en el conocimiento, sino que se logra una docencia de calidad, ya que la mejor forma de aprender es investigando. En el caso de los alumnos, en el nuevo escenario universitario la investigación ya no se reserva sólo para el tercer ciclo (doctorado), también se inicia a los de los primeros ciclos (diplomatura y licenciatura) aunque sea a un nivel muy elemental, en las técnicas que pueden formarles para investigar en el futuro: búsqueda de información, etc.

Por otra parte, afectada substancialmente por la globalización y las nuevas tecnologías, la investigación está acentuando progresivamente su carácter internacional y cooperativo.

\section{La biblioteca universitaria y su adaptación al nuevo contexto universitario}

La biblioteca universitaria, como una pieza clave de la universidad, sin duda alguna va a verse afectada por los cambios que representa el nuevo modelo de universidad.

Los cambios del sistema universitario van a requerir una biblioteca más activa, como servicio de apoyo a la docencia, la investigación y el aprendizaje, las misiones más significativas de la universidad. Por otra parte, el papel más activo de apoyo a las misiones de la universidad, requieren una mayor identificación de la biblioteca con aquella, en nuestra opinión, dos son las formas más importantes para ello: una mayor integración de la biblioteca en el marco institucional y un aumento de la preocupación por la calidad en la gestión, al constituir esta última, como ya hemos señalado, la nota más relevante del nuevo modelo de universidad.

Ante las nuevas exigencias ha surgido un modelo de biblioteca definido como Centro de Recursos para el Aprendizaje y la Investigación (CRAI).

\subsection{Integración en el marco institucional}

La razón de ser de la biblioteca universitaria es servir de apoyo a la universidad en el desarrollo de sus funciones. Para que resulte eficaz, debe existir una integración entre ella y la misión, fines y objetivos de la universidad.

Sin embargo, una de las debilidades que presentan algunas bibliotecas es la falta de integración real en la universidad a la que pertenecen, extremo relacionado con el escaso peso que ejercen dentro de la institución. En España, el poco peso que demuestran está relacionado con los métodos docentes aplicados tradicionalmente, basados fundamentalmente en la clase magistral. Los alumnos, hablando de forma general, son sujetos pasivos que aprenden asistiendo a clase y estudiando apuntes y, como mucho, la bibliografía recomendada por el profesor.

"Los cambios del sistema universitario van a requerir una biblioteca más activa como servicio de apoyo a la docencia, la investigación y al aprendizaje"

En este método de enseñanza la biblioteca tiene muy poco peso en la universidad porque los alumnos la utilizan en gran medida como una sala para estudiar apuntes.

No obstante, la concepción anglosajona que puede resumirse en la frase, ya clásica: "la biblioteca es el corazón de la universidad", ha ido haciendo escuela y paulatinamente distintos colectivos universitarios y de la administración han trabajado para lograr que la biblioteca fuera algo más que una sala donde estudiar apuntes. A título de ejemplo, en España hay que aludir al papel dinamizador de Rebiun.

Sin duda alguna, el nuevo método de aprendizaje que supone la implantación del EEES, contribuirá a lograr una mayor integración y peso de la biblioteca en las universidades, al basarse en una mayor participación del estudiante en el proceso de aprendizaje y requerir más utilización de recursos y servicios bibliográficos.

A nivel nacional, la integración de las bibliotecas universitarias en sus respectivas universidades se impulsa a través de la integración de Rebiun en la Conferencia de Rectores de las Universidades Españolas (CRUE), como una de las mesas sectoriales que constituyen dicha conferencia. Ya a nivel individual, son varios los canales a través de los cuales puede integrarse en la universidad. De ellos, citamos algunos ejemplos:

- La normativa de las universidades, a través de la cual se regula su funcionamiento. La normativa de más categoría son los estatutos en los que las universidades definen el papel que la biblioteca juega.

- Los planes estratégicos de las universidades, que plantean el logro de una serie de objetivos en determinados plazos. La biblioteca debe integrarse y participar de ellos, algo que debe reflejarse claramente en el correspondiente plan y difundirse 
entre toda la comunidad universitaria.

- El porcentaje del presupuesto global de la universidad asignado a la biblioteca, evidencia la importancia que tiene para su correspondiente universidad y el grado de integración en la misma. No hay que olvidar que de la asignación presupuestaria ordinaria van a depender sus recursos básicos: colecciones, personal, instalaciones. A esto, como señala Rebiun (1997, p. 62 ), hay que añadir que la biblioteca debe estar incluida en el plan de inversiones de la universidad.

- Una integración real requiere también la participación activa de la biblioteca en los diferentes órganos de gobierno de la universidad. Recíprocamente, los distintos estamentos deben tomar parte en las decisiones que afectan a los principales temas relacionados con los usuarios, a través de su participación en las comisiones de biblioteca.

- La biblioteca universitaria debe evolucionar para armonizar sus servicios con las nuevas necesidades que genera el nuevo sistema de aprendizaje, en el que las nuevas tecnologías son decisivas. Ello requerirá una mayor integración con los distintos servicios de la universidad: administrativos, informáticos, etc. (Winkworth, 2001, p. 148). La mayor integración de la biblioteca en la universidad supondrá una progresiva participación en proyectos transversales con los servicios de informática, personal docente, etc. En este punto es preciso recordar su función emergente como editora de materiales digitales relacionados con la docencia y la investigación.

- Son necesarios canales formales e informales de comunicación eficaces entre la biblioteca y la comunidad universitaria y viceversa. A través de ellos se difunden noticias, nuevos servicios, etc. y la comunidad universitaria, hace llegar a la biblioteca peticiones, preguntas, expone quejas, etc.

\subsection{La búsqueda de calidad} en la gestión

Como ya hemos señalado, uno de los retos con que se enfrenta la universidad española es la búsqueda de calidad en la gestión para poder competir tanto entre las distintas universidades españolas como con otras europeas y de otras partes del mundo. Por ello, la biblioteca universitaria, como uno más de los servicios de la universidad, también está obligada a buscar la calidad en su gestión. Igual que la universidad, tiene que demostrar que los recursos recibidos son bien gestionados y se traducen en servicios de calidad, entendiendo por calidad la adecuación a los objetivos.

"La biblioteca universitaria, como uno más de los servicios de la universidad, también está obligada a

\section{buscar la calidad en su gestión"}

La preocupación por llevar a cabo una gestión de calidad en el mundo bibliotecario no es nueva, como pone de manifiesto la antigüedad de algunos estudios sobre evaluación del rendimiento. Como ejemplo podemos señalar que Philip K. Morse publicó su obra: Library effectiveness: a systems approach en 1968. La novedad es que en este momento, la evaluación de los servicios bibliotecarios se sitúa en el contexto de la universidad. La biblioteca es uno de sus servicios y como tal debe ser evaluado, y debe rendir cuentas a su universidad. En este entorno, se está extendiendo la idea de que los presupuestos deben condicionarse a la búsqueda de calidad i las bibliotecas reciben subvenciones en premio a la calidad. En la línea de integración a la que nos referíamos al principio de este apartado, es la Aneca, que tiene la misión de evaluar la calidad de la educación superior, la que también se encarga de otorgar el certificado de calidad de las bibliotecas universitarias.

\subsection{La biblioteca universita-} ria como Centro de Recursos para el Aprendizaje y la Investigación (CRAI)

Ya se ha señalado antes que en este contexto nace el CRAI como nuevo modelo de biblioteca. Rebiun presenta este modelo en su Plan Estratégico 2003-2006, donde señala la necesidad de pasar de la biblioteca como centro de soporte a la docencia, a la biblioteca como Centro de Recursos para el Aprendizaje y la Investigación (CRAI), es decir, de una biblioteca con un papel pasivo, reactivo y no participativo, a otra activa y participativa en el aprendizaje, la docencia y la investigación.

El CRAI es un lugar físico donde tanto profesores como alumnos puedan encontrar información, asesoramiento y ayuda para utilizar tecnologías informáticas, multimedia, etc. como medios necesarios para su actividad en la universidad. Ello supone la formación de un equipo de profesionales tales como informáticos, técnicos audiovisuales, asesores de pedagogía, etc., que trabajan juntos. De esta forma, se convierte en una pieza clave en la planificación de un sistema de información único para toda la universidad y permite el desarrollo de proyectos transversales. La biblioteca debe formar parte activa del $C R A I$, en el que se integra junto con el servicio de informática y otros de la universidad.

En su II Plan Estratégico 20072010, Rebiun se plantea como primer objetivo seguir apoyando el desarrollo de las bibliotecas universitarias concebidas de esta manera. 


\section{Hacia una nueva definición de biblioteca universitaria}

A modo de conclusión y basándonos en lo anteriormente expuesto, a continuación proponemos una definición de biblioteca universitaria: Es un centro de recursos de información que basa su gestión en la filosofía de la globalización, en el uso de las nuevas tecnologías de la información y de la comunicación, en la cooperación y en la calidad. Tiene como principal misión proporcionar a la comunidad universitaria todos aquellos servicios, documentos y recursos informativos propios o ajenos, necesarios para que aquella desarrolle con eficacia sus funciones docentes, de investigación y aprendizaje.

\section{Bibliografía}

García Camarero, E.; García Melero, L. La biblioteca digital. Madrid: Arco Libros, 2001. ISBN 84-7635-486-X.
Giordano, T. "Library consortium models in europe: a comparative análisis". En: Alexandria, 2000, v. 14, n. 1, pp. 41-52.

Herring, Mark Y. "10 reasons why the internet is no substitute for a library". En: American libraries, 2001, April, pp. 76-78.

Miguel, J. M. de; Caïs, J.; Vaquera, E. Excelencia: calidad de las universidades españolas Madrid: Centro de Investigaciones Sociológicas, 2001. ISBN 84-7476-313-4

Morse, P. K. Library effectiveness: a systems approach. Cambridge, Massachusetts: M.I.T Press, 1968

Moscoso, P. La nueva misión de las bibliotecas universitarias ante el Espacio Europeo de Enseñanza Superior. Consultado en: 10-01-07.

http://biblioteca.uam.es/documentos/Jornadas_ REBIUN/4\%20-\%20nueva_mision_bibliotecas. $p d f$

Neouze, V. "La numérisation dans les Bibliothèques de 1'Enseignement Supérieur en France". En: Liber quarterly, 2002, v. 12, n. 4, pp. 344 352.

Orera Orera, L. "La evolución en la formación de los bibliotecarios". En: Documentación de la ciencias de la información, 2002, v. 25, pp. 167188.

Orera Orera, L. "La edición digital de tesis doctorales: hacia la resolución de los problemas de accesibilidad". En: Revista interamericana de bibliotecología, 2003, v. 26, n. 1, pp. 11-35.

Orera Orera, L. (ed.). La biblioteca universitaria: análisis en su entorno híbrido. Madrid: Síntesis, 2005. ISBN 84-9756-349-2.

Pérez Gómez, M. A.; Cadavid Arango, C. A. "Desarrollo de colecciones propias vs. acceso remoto". En: Primer seminario internacional sobre desarrollo de colecciones, 1997, pp. 177-197.

Rebiun. Normas y directrices para bibliotecas universitarias y científicas. Madrid: Dirección General del Libro, Archivos y Bibliotecas, 1997.

Rebiun. Plan estratégico 2003-2006. Consultado en: 20-02-07.

http://www.crue.org/rebiun/PlanEstrategico.pdf

Rebiun. II Plan estratégico 2007-2010. Consultado en: 20-02-07.

http://www.crue.org/pdf/Pto.\%208.\%20II\%20P LAN\%20ESTRAT\%C3\%89GICO\%20REBIUN \%202007-2010.pdf

Winkworth, I. "La biblioteca universitaria híbrida". En: Boletín de la Asociación Española de Archiveros, bibliotecarios, Museólogos y Documentalistas (Anabad), 2001, n. 2, pp. 139-149.

Luisa Orera-Orera, Universidad de Zaragoza.

mlorera@unizar.es

Via Benedetto da Maiano 3

50014 Fiesole (Florencia)

Italia

Tel. +3905550181

Fax +39055 5018201

info@casalini.it

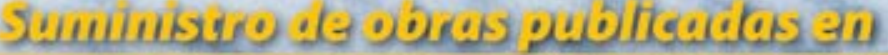

\section{Sirvicios fitegrados para la biblioteca}

\section{Libros}

Revistas

Approval Plan

Registros MARC

Bases de datos en línet razó

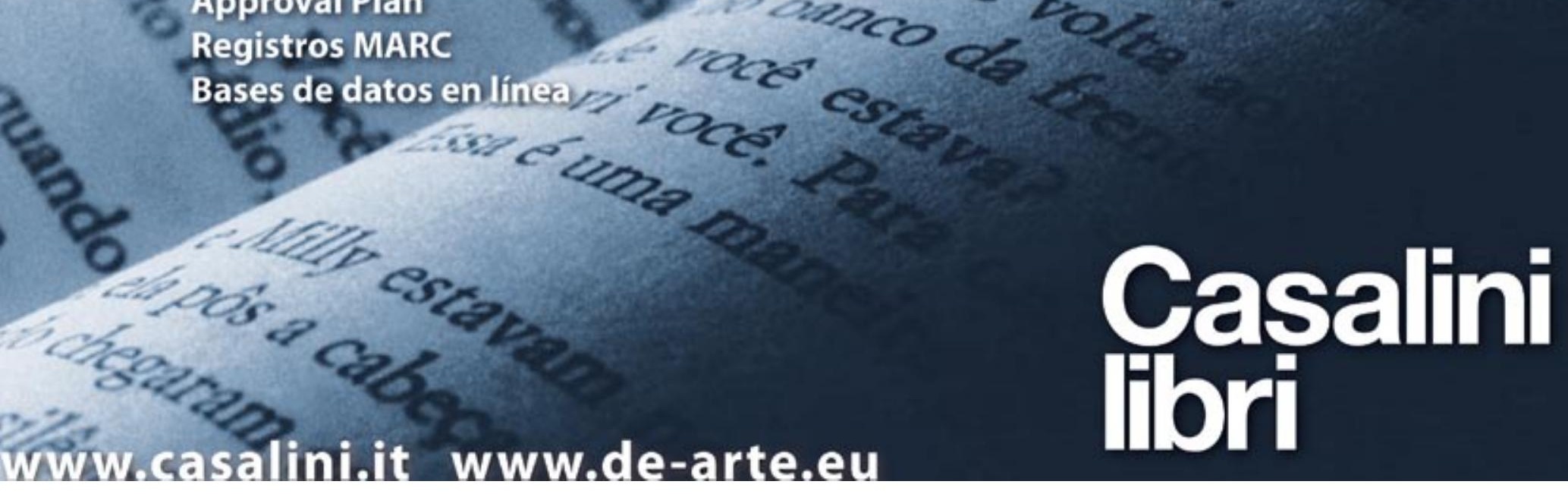

